\title{
Article
}

\section{Which jump-landing task best represents lower extremity and trunk kinematics of unanticipated cutting maneuver?}

Hanzlíková, Ivana, Richards, James, Athens, Josie and HébertLosier, Kim

Available at http://clok.uclan.ac.uk/36759/

Hanzlíková, Ivana, Richards, James ORCID: 0000-0002-4004-3115, Athens, Josie and Hébert-Losier, Kim (2021) Which jump-landing task best represents lower extremity and trunk kinematics of unanticipated cutting maneuver? Gait \& Posture, 85 . pp. 171-177. ISSN 0966-6362

It is advisable to refer to the publisher's version if you intend to cite from the work. http://dx.doi.org/10.1016/j.gaitpost.2021.02.003

For more information about UCLan's research in this area go to http://www.uclan.ac.uk/researchgroups/ and search for <name of research Group>.

For information about Research generally at UCLan please go to http://www.uclan.ac.uk/research/

All outputs in CLoK are protected by Intellectual Property Rights law, including Copyright law. Copyright, IPR and Moral Rights for the works on this site are retained by the individual authors and/or other copyright owners. Terms and conditions for use of this material are defined in the policies page.

\section{CLoK}

Central Lancashire online Knowledge www.clok.uclan.ac.uk

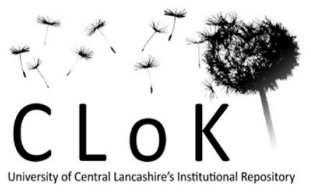




\title{
Which jump-landing task best represents lower extremity and trunk kinematics of unanticipated cutting maneuver?
}

\author{
Ivana Hanzlíková, Jim Richards, Josie Athens, Kim Hébert-Losier
}

\begin{abstract}
Background: The double-leg jump-landing (DLJL) task is commonly used as a movement screen that can be implemented in large cohorts of athletes. However, it is debatable whether the DLJL is ecologically valid and reflects sporting requirements or injury-prone situations, such as cutting and pivoting.
\end{abstract}

Research question: Which jump-landing movement variation best represents the kinematics of unanticipated side-step cutting?

Methods: Forty-two participants (25 males and 17 females) performed unanticipated side-step cutting and four jump-landing tasks: DLJL, rotated DLJL (DLJL ${ }_{\text {rot }}$ ), single-leg jump-landing (SLJL), and rotated SLJL (SLJL ${ }_{\text {rot }}$ ). Ankle, knee, hip, pelvis, and trunk angles and angular velocities, and pelvic linear accelerations were collected at initial contact and during the first 100 milliseconds after initial contact (minimum, maximum, and range values) using a threedimensional infrared camera system and inertial measurement units. Pre-contact foot-ground angles and subjective task difficulty ratings were also recorded. Intraclass correlation coefficients (ICC) between cutting and jump-landing kinematics were calculated for each participant and jump-landing variation. Friedman tests with pairwise comparisons were then used to compare the degree of association between the four different jump-landing tasks at the specified time events and to compare the difficulty ratings.

Results: Considering the ICC values across the events of interest, the kinematics of the DLJL were the least associated with those of cutting ( ICC $=0.00$ to 0.81 ), and DLJL $L_{\text {rot }}(\mathrm{ICC}=0.34$ 
to 0.81 ) and $\mathrm{SLJL}_{\text {rot }}$ (ICC $=0.31$ to 0.80 ) biomechanics the most. Participants rated the perceived challenge of the single-leg tasks in a similar manner to cutting ( $p>0.103)$, and the SLJL $L_{\text {rot }}$ as the most difficult task (median = "neutral", mode = "neutral").

Significance: Due to their biomechanical associations with cutting maneuver and subjectivelyrated difficulty levels, both DLJL $\mathrm{L}_{\text {rot }}$ and SLJL $\mathrm{S}_{\text {rot }}$ may be more appropriate and ecologically valid for screening for risk of injury across a range of sports.

Keywords: Injury risk screening, ACL, single-leg landing, rotated single-leg landing, doubleleg landing, rotated double-leg landing

Declarations of interest: none

Word count: 3000 words

\section{Highlights:}

- DLJL kinematics were the least related to unanticipated cutting kinematics.

- Rotated tasks were the most strongly related to unanticipated cutting kinematics.

- Difficulty ratings of single-leg tasks were similar to unanticipated cutting.

- SLJL $L_{\text {rot }}$ was subjectively rated as the most difficult task among the tasks tested.

- Rotated tasks may be more ecologically valid than DLJL for injury risk screening. 


\section{Introduction}

The double-leg jump-landing (DLJL) task is commonly used to evaluate landing biomechanics in research and clinics [1-5], and can be implemented as a movement screen in large cohorts. However, the DLJL has limitations, including that it does not fully represent movements associated with high risk of injury in a sporting context. Athletes frequently land on one leg and injuries often involve complex movements, such as side-cutting, pivoting, or cross-cutting [6]. Krosshaug et al. [3] criticized using the DLJL as a screening task to predict anterior cruciate ligament (ACL) injury in sports, given that it is not challenging enough or reflective of common sport movements. Moreover, it has been shown that sport medicine and coaching professionals are unable to correctly identify athletes who subsequently sustain an ACL injury through visual assessment of DLJL kinematics [5].

The Landing Error Screening System (LESS) is a popular injury-risk screening tool that uses the DLJL [7]. Reliability and validity of the LESS has been established, but its predictive value is unclear [7]. The inconsistent findings relating to its predictive value may be due to the non-sport specific nature of the LESS task, supporting that the DLJL task is not challenging enough to unveil 'risky' movement patterns [4]. In examining the efficiency of the LESS to identify high-risk lower extremity mechanics during a sport-specific landing task associated with ACL injury in netball, Fox et al. [8] concluded that the LESS might have low applicability in identifying netballers at high injury risk.

In recent years, other ACL injury-risk clinical screening tools have been proposed, including the Cutting Movement Assessment Score (CMAS) [9]. This tool identifies potentially high-risk movement patterns linked with greater knee valgus moments during sidestep cutting [9]. The CMAS has been shown reliable and valid against three-dimensional (3D) motion capture [9]. However, its predictive value for injury has not been established, and the 
space and time requirements for testing are greater compared to the LESS, and may therefore be less suitable for large-scale screening [9].

There is a need for injury screening methods based on more sport-related and injuryrelated tasks, whilst keeping the screening task viable for large-scale screening initiatives. Incorporating single-leg landing and rotational movements within injury screening models may offer an appealing alternative to DLJL. Non-contact lower-extremity injuries, including to the ACL, result from poor whole-body movement control in all three planes of motion, rather than dysfunction or altered movement in a single joint or plane of motion [10]. Therefore, rather than considering discrete kinematic measures at specific joints, our aim was to compare the level of association between whole-body kinematics of four jump-landing tasks to a sportspecific and injury-specific unanticipated side-step cutting maneuver. The jump-landing task demonstrating the strongest association with cutting maneuver may be suitable for large-scale injury-risk screening in sports that involve cutting (e.g., soccer, field hockey) or a mix of cutting and jump-landing (e.g., netball, handball) tasks. Additionally, subjective ratings of the difficulty of each task were examined. We hypothesized that single-leg jump-landing (SLJL) would show the strongest correlations to the side-step cutting maneuver, and that SLJL rot $_{\text {Would }}$ be rated as the most difficult.

\section{Methods}

\subsection{Sample size estimation}

A two-tailed hypothesis using an $80 \%$ power $(\beta=0.20), 5 \%$ significance level $(\alpha=$ $0.05)$, critical values of the $t$-distribution, and data from previous studies using a similar 3D motion capture set-up and marker set were used to determine the sample size $[11,12]$. Given the absence of correlation data for sample size estimations, values reporting knee flexion at initial contact (IC), coronal plane knee range of motion, and transverse plane knee range of 
motion were compared between the SLJL and side-step cutting tasks. It was anticipated that detecting differences between these two tasks would require the largest sample size. This analysis indicated that 33 participants were needed to identify differences between these two tasks. To account for $25 \%$ withdrawals or missing data, 42 participants were recruited.

\subsection{Participants}

Inclusion criteria were: age between 16 and 35 years, free from any injury or illness that prohibited or limited physical activity participation, and regular participation in a team sport that involved cutting. A history of injury or surgery was not an exclusion criterion given that injury-risk screening is relevant to previously injured athletes. A Health Research Ethics Committee approved the study protocol [HREC(Health)2018\#27], which adhered to the Declaration of Helsinki. All participants signed a written informed consent document before participating that explained the potential risks.

\subsection{Experimental procedure}

Participants were familiarized with the experimental protocol and all testing was completed in one session. After completing a baseline questionnaire and the International Physical Activity Questionnaire [13], participants performed five tasks: 1) double-leg jumplanding (DLJL); 2) rotated double-leg jump-landing (DLJL $\left.{ }_{r o t}\right) ; 3$ ) single-leg jump-landing (SLJL); 4) rotated single-leg jump-landing (SLJL rot $_{\text {) }}$; and 5) unanticipated side-step cutting. The DLJL followed the LESS protocol [14], requiring participants to jump forward from a 30$\mathrm{cm}$ high box with both feet, landing to a distance equal to half of their body height, and then immediately jump upwards for maximal height. For DLJLrot, the protocol was similar to DLJL, but participants rotated $90^{\circ}$ in the air before landing on both legs (Figure 1). For SLJL, the protocol was similar to DLJL, but landing was on one leg (Figure 1). For SLJLrot, the protocol was similar to DLJL $L_{\text {rot, }}$ but landing was on one leg (Figure 1). To begin SLJL and SLJLrot tasks, 
participants stood on one leg. Due to the difficulty of these tasks, the landing distance was reduced to $25 \%$ of body height.

For the unanticipated side-step cutting, participants started five meters from the target cutting area. When participants moved within the target area, timing gates (Swift Performance SpeedLight ${ }^{\mathrm{TM}}$ ) triggered one of two pairs of lights to signal the cutting direction. During cutting, participants were required to remain between two lines taped to the floor, indicating a cutting angle of $60^{\circ}$ to $90^{\circ}$. A minimum approach speed of $3.5 \mathrm{~m} / \mathrm{s}$ at the penultimate foot contact was required based on previous studies to mimic a typical game setting [15]. Any trials performed at slower speeds were disregarded and repeated.

The testing order was randomized for task and then direction (i.e., left or right). After a familiarization period of typically two attempts per task, each participant completed three successful repetitions. With the exception of DLJL, all tasks were performed three times to the left and three times to the right. The Perceived Recovery Status Scale [16] was used to ensure sufficient subjective recovery between trials and tasks, with participants needing to self-report ratings $\geq 7$ before proceeding to the next trial or task. On average, time between trials was 30 seconds, and between tasks was 3 minutes. Furthermore, after each task, participants were asked to evaluate the level of difficulty of the task using the following 5-point Likert scale: 1very difficult, 2 - difficult, 3 - neutral, 4 - easy, 5 - very easy.

\subsection{Instrumentation}

Whole-body motion was recorded at $200 \mathrm{~Hz}$ during all five tasks using an 8-camera 3D motion capture system (Oqus 700+ cameras) and software (Qualisys Track Manager v.2019.1, Qualisys AB, Gothenburg, Sweden). Forty-two 12.5-mm retroreflective markers and five clusters were taped onto the skin and shoes, which were modelled using the Calibrated Anatomical System Technique [17]. An additional cluster was placed on the right side of the 
pelvis to improve segment tracking (Figure 2). Three inertial measurement unit (IMU) sensors (Delsys Trigno IM sensors, Delsys Inc., MA, USA) sampling at $148 \mathrm{~Hz}$ were synchronized with the 3D motion capture system to assist with event determination in the absence of force plates. Two sensors were placed bilaterally $4 \mathrm{~cm}$ above the lateral malleoli, and one attached over the sacrum.

\subsection{Data processing}

Data were exported to .c3d format and processed using Visual3D Professional ${ }^{\mathrm{TM}}$ (v.6.01.36, C-Motion Inc., Germantown, Maryland, USA). A 13 rigid segment biomechanical model with six degrees of freedom at each joint was constructed. The local coordinates of all segments were derived from a static trial captured prior to the dynamic trials. Any marker data gaps less than 10 frames were interpolated using a third order polynomial fit algorithm. A fourth order low-pass Butterworth filter with a cut-off frequency of $15 \mathrm{~Hz}$ was then applied to the marker data [18]. IMU data were visually assessed using a range of cut-off frequencies (15 to $100 \mathrm{~Hz}$ ), and $80 \mathrm{~Hz}$ was confirmed as the best at preserving all high-frequency signal characteristics, while also removing noise. The sacrum IMU acceleration data were corrected based on the pelvis angle in all three planes.

Kinematic parameters were calculated using an $\mathrm{XYZ}$ cardan sequence, equivalent to the joint coordinate system [19]. Ankle, knee, hip, pelvis, and trunk angles and angular velocities, and pelvic linear accelerations were extracted at IC and from the 100 milliseconds after IC (minimum, maximum, and range values). Additionally, foot-ground angles in all three planes were extracted one frame before IC to explore pre-landing strategies [20]. IC was defined based on the peak vertical acceleration from IMU sensors placed above the lateral malleoli for jumplanding tasks, and as the instance when the cutting-leg foot center of gravity acceleration in the vertical plane (i.e., plane perpendicular to the floor) of the lab coordinate system $(z)$ reached a 
maximum value for the cutting task. The 100-millisecond timeframe was chosen as ACL injuries have been reported to occur within this period [21]. For DLJL, data from the pelvis, trunk, and both extremities were extracted. For DLJLrot, data from the pelvis, trunk, and the extremity furthest away from the box were extracted. For single-leg tasks, data from the pelvis and trunk from the landing extremities were extracted. The directionality and interpretation of joint movements are presented in Table 1 . Furthermore, the pelvis center of gravity velocity at IC and cutting angle during the cutting maneuver were extracted to quantify cutting performance.

\subsection{Statistical analysis}

Joint angle, angular velocity, and IMU data from the three trials of each task were averaged for statistical analyses. To determine which jump-landing task was the most reflective of the sport-specific unanticipated cutting maneuver, the association between the kinematic variables extracted ( $n=72$, Supplementary data) during cutting and each of the jump-landing tasks was quantified using single measurement, consistency agreement, two-way random effect intraclass correlation coefficients (ICCs) for each participant [22]. Both dominant and nondominant lower extremities were included in the analysis to derive the ICC for each participant.

Subsequently, Friedman tests with Wilcoxon signed-rank tests during post-hoc comparisons were used to compare the strength of the associations of the kinematic variables from the different jump-landing tasks to the cutting task at IC (including pre-landing footground angles) and during the 100 milliseconds after IC, Table 1. Friedman tests were used due to violated assumptions for parametric testing [23]. Subjective ratings regarding task difficulty were described using median, mode, and frequency indicators, and compared between tasks using the Friedman test with Wilcoxon signed-rank tests during post-hoc comparisons. The significance level was set at $p \leq 0.05$ for all analyses, which were performed 
using Microsoft ${ }^{\circledR}$ Excel (Office 365 MSO) and RStudio ${ }^{\circledR}$ (version 1.1.463) with R (version 3.5.2).

\section{Results}

Forty-two participants (25 males and 17 females) volunteered. Age, height, and mass (mean \pm standard deviation) for males were $23.6 \pm 4.1$ years (range 17 to 32 years), $182.2 \pm$ $6.4 \mathrm{~cm}$, and $85.0 \pm 11.9 \mathrm{~kg}$; and for females were $22.2 \pm 5.7$ years (range 16 to 35 years), 169.1 $\pm 6.0 \mathrm{~cm}$, and $63.7 \pm 6.8 \mathrm{~kg}$. Ninety-three percent of participants were right-leg dominant based on the preferred leg to kick a ball. The International Physical Activity Questionnaire indicated that activity levels were high, moderate, and low in $60 \%, 38 \%$, and $2 \%$ of participants, respectively. Thirty-one percent of participants played soccer, $26 \%$ rugby, $17 \%$ ultimateFrisbee, 14\% netball, 7\% basketball, and 5\% field hockey. Participants' level of engagement with sport was 55\% club level, $21 \%$ recreational, $17 \%$ national level, and $7 \%$ school level. Participants were involved in physical activity 3 times per week (median) for on average $6.7 \pm$ 4.4 hours weekly. On average, our sample had participated in physical activity on a regular basis for $10.5 \pm 6.2$ years. In all analyses, there were no missing data.

Mean values and standard deviations of all extracted variables are presented as Supplementary data. Overall, the mean cutting angle was $58.3 \pm 9.8^{\circ}$ and cutting speed at IC was $3.4 \pm 0.5 \mathrm{~m} / \mathrm{s}$. At IC, rotated tasks were more strongly $(p<0.001)$ associated with cutting kinematics than non-rotated tasks based on ICCs (Figure 3A). The minimum values of the explored variables during all jump-landing tasks showed similar levels of associations to those of cutting, with mean ICC values $\geq 0.66$ for all tasks (Figure 3B). The maximum values of the kinematic variables during the DLJL $\mathrm{L}_{\text {rot }}$ was the most strongly associated with cutting compared to all other jump-landing tasks (ICC 0.74, $p<0.001$ ), and DLJL and SLJL the least associated (Figure 3C). The range of motion in all jump-landing tasks showed similar levels of association 
to those of cutting, with mean ICC values $\geq 0.80$ for all tasks (Figure 3D). Overall, when considering ICC values across the events of interest, the DLJL kinematics appeared to be the least associated with cutting, and DLJL $L_{\text {rot }}$ the most followed by SLJL rot. $_{\text {. }}$

Additionally, subjective ratings relating to task difficulty significantly differed between tasks $(p<0.022)$, expect for between cutting and SLJL $(p=1.000)$, cutting and $\operatorname{SLJL}_{\text {rot }}(p=$ 0.103), and SLJL and $\operatorname{SLJL}_{\text {rot }}(p=0.052)$. Participants rated the DLJL as the easiest task to perform (median = "easy", mode = "very easy"), and the SLJL ${ }_{\text {rot }}$ as the most difficult (median $=$ "neutral", mode = "neutral"). Five percent of participants rated the SLJL $L_{\text {rot }}$ as "very difficult" and $31 \%$ as "difficult", Figure 4.

\section{Discussion}

Almost two decades ago, Hewett et al. [1] suggested the DLJL could be used to screen for risk of ACL injury. The DLJL has become commonplace in the assessment of landing biomechanics. However, several studies have criticized the DLJL task, stating it is not reflective of common sport movements and injurious situations, not challenging enough, and poor for predicting ACL injury [3-5, 8]. Our results support these statements and indicate the lowest association between DLJL and sport-specific cutting kinematics when compared to other jump-landing tasks. Furthermore, according to the subjective ratings, DLJL was rated as easy $(n=19,45 \%)$ and very easy $(n=20,48 \%)$, reflecting the low perceived challenge of this task. From the tasks tested, the DLJL ${ }_{\text {rot }}$ showed the greatest biomechanical similarities to cutting based on ICC values, followed by SLJL rot. Furthermore, single-leg landing tasks had a similar perceived challenge than cutting, with SLJL ${ }_{\text {rot }}$ subjectively rated as the most difficult. Therefore, the two rotated jump-landing tasks (DLJL $\mathrm{L}_{\text {rot }}$ and $\mathrm{SLJL}_{\mathrm{rot}}$ ) may be more appropriate than the DLJL to reveal risky movement patterns that are more sport-specific and challenging. 
Overall, single-leg landings are biomechanically more challenging for the knees than double-leg landings, with lower knee flexion at IC, lower sagittal plane knee displacement, greater frontal plane knee displacement, and greater knee abduction moments [24]. Single-leg landings are also more common in sports and during injury situations than double-leg landings [21, 25]. Most athletic movements involve unilateral propulsion or stabilization (e.g., running, kicking, jumping). Video analysis of injury situations during games show that up to $80 \%$ of non-contact ACL injuries occur during single-leg landings or cuttings [21, 25]. Moreover, high injury-risk movement patterns may become more apparent during single-leg landings due to greater lower-extremity loadings, smaller bases of support, and greater motor control challenges to stabilize the pelvis and trunk [26]. All of these variables probably contributed to the greater perceived challenge of single-leg tasks in our study. Altogether, our findings suggest that single-leg tasks may more accurately reflect the challenge associated with unanticipated cutting. However, compared to SLJL, SLJL ${ }_{\text {rot }}$ showed stronger associations with cutting biomechanics and was rated as the most difficult task to perform. Hence, compared to SLJL, SLJL $_{\text {rot }}$ may be better suited to reveal movement patterns present during more challenging sport situations and, in turn, have a greater association with injury-risk profiles specific to ACL injuries.

Due to their subjectively-rated difficulty levels and biomechanical association with cutting movements, both DLJL ${ }_{\text {rot }}$ and SLJLrot may be more appropriate screening tasks for landing (e.g., volleyball and basketball) and cutting (e.g., soccer, netball, field hockey, handball, American football, and rugby) sports than the traditional DLJL. Given that twodimensional video assessments of double-leg and single-leg landings have been used to identify athletes with increased risk of non-contact knee injuries $[2,27]$ and both tasks require minimal space requirements, they could be useful for large-scale screening initiatives. However, establishing what specific parameters from the DLJL $\mathrm{rot}_{\text {or }} \mathrm{SLJL}_{\text {rot }}$ may be useful in the clinical 
assessment of injury risk requires further research, and prospective studies are needed to confirm the psychometric properties and predictive value of these tasks.

Noteworthy is that our study examined the association between kinematic variables, and not their comparability. The concept of kinetic chains stipulates that each joint movement and underlying muscle contraction are coupled with movements and muscle contractions in other joints [28]. For example, trunk control is closely related to the ability of the hip and pelvis to adequately respond to unexpected movements and forces generated by distal body segments [29]. For instance, weak hip abductors lead to contralateral pelvis drop (Trendelenburg position); to compensate for a Trendelenburg position, the trunk inclines laterally towards the stance leg and produces a greater lateral lever arm relative to the knee joint centre and increases the knee valgus moment and ACL strain [29]. Hence, every joint movement in each plane may contribute to non-contact lower-extremity injuries, supporting that whole-body movement patterns and control should be considered when screening for injury risk. Therefore, rather than comparing specific angles in given joints or planes of motion, this study examined the association between whole-body movement patterns during cutting and different jump-landing variations. Given that cutting was used as the sport-specific task to determine the relevance of various jump-landing movements to screen for potential risk of ACL injuries, our results might be of greater relevance for athletes and sports that involve cutting (e.g., soccer, field hockey) or cutting and jump-landing (e.g., netball, handball) rather than predominantly jump-landing (e.g., volleyball). Moreover, due to absence of force plates, we were unable to compute joint moments through inverse dynamics, which could have provided further insight into the biomechanical associations between the tasks tested.

\section{Conclusion}


Within the tasks explored, whole-body kinematics of DLJL ${ }_{\text {rot }}$ were the most strongly and consistently associated with cutting kinematics, followed by SLJL rot. The SLJL $\mathrm{L}_{\text {rot }}$ task was rated as the most difficult to perform and had similar self-reported difficulty levels to cutting. Therefore, rotated jump-landing tasks may be more appropriate than the DLJL to reveal risky movement patterns present during rapid changes of direction and landing, which could be implemented in large-scale screening as an alternative to DLJL.

\section{Funding sources}

This research did not receive any specific grant from funding agencies in the public, commercial, or not-for-profit sectors.

\section{Conflict of interest statement}

We can confirm that there is no conflict of interests for any of the authors.

\section{Acknowledgement}

There are no acknowledgements to make. 


\section{References}

[1] T.E. Hewett, G.D. Myer, K.R. Ford, R.S. Heidt Jr, A.J. Colosimo, S.G. McLean, et al., Biomechanical measures of neuromuscular control and valgus loading of the knee predict anterior cruciate ligament injury risk in female athletes: a prospective study, Am. J. Sports Med. 33(4) (2005) 492-501.

[2] D.A. Padua, L.J. DiStefano, A.I. Beutler, S.J. de la Motte, M.J. DiStefano, S.W. Marshall, The Landing Error Scoring System as a screening tool for an anterior cruciate ligament injury-prevention program in elite-youth soccer athletes, J. Athl. Train. 50(6) (2015) 589-595.

[3] T. Krosshaug, K. Steffen, E. Kristianslund, A. Nilstad, K.-M. Mok, G. Myklebust, et al., The vertical drop jump is a poor screening test for $A C L$ injuries in female elite soccer and handball players: a prospective cohort study of 710 athletes, Am. J. Sports Med. 44(4) (2016) 874-883.

[4] H.C. Smith, R.J. Johnson, S.J. Shultz, T. Tourville, L.A. Holterman, J. Slauterbeck, et al., A prospective evaluation of the Landing Error Scoring System (LESS) as a screening tool for anterior cruciate ligament injury risk, Am. J. Sports Med. 40(3) (2012) 521-526.

[5] A.I. Mørtvedt, T. Krosshaug, R. Bahr, E. Petushek, I spy with my little eye... a knee about to go 'pop'? Can coaches and sports medicine professionals predict who is at greater risk of ACL rupture?, Br. J. Sports Med. 54(3) (2020) 154-158.

[6] J.M. Hootman, R. Dick, J. Agel, Epidemiology of collegiate injuries for 15 sports: summary and recommendations for injury prevention initiatives, J. Athl. Train. 42(2) (2007) 311-319.

[7] I. Hanzlíková, K. Hébert-Losier, Is the Landing Error Scoring System reliable and valid? A systematic review, Sports Health 12(2) (2020) 181-188.

[8] A.S. Fox, J. Bonacci, S.G. McLean, N. Saunders, Efficacy of ACL injury risk screening methods in identifying high-risk landing patterns during a sport-specific task, Scand. J. Med. Sci. Sports 27(5) (2017) 525-534.

[9] T. Dos'Santos, A. McBurnie, T. Donelon, C. Thomas, P. Comfort, P.A. Jones, A qualitative screening tool to identify athletes with 'high-risk' movement mechanics during cutting: the cutting movement assessment score (CMAS), Phys. Ther. Sport 38 (2019) 152-161.

[10] T.E. Hewett, K.R. Ford, B.J. Hoogenboom, G.D. Myer, Understanding and preventing ACL injuries: current biomechanical and epidemiologic considerations-update 2010, N. Am. J. Sports Phys. Ther. 5(4) (2010) 234-251.

[11] I. Hanzlíková, J. Richards, M. Tomsa, A. Chohan, K. May, D. Smekal, et al., The effect of proprioceptive knee bracing on knee stability during three different sport related movement tasks in healthy subjects and the implications to the management of Anterior Cruciate Ligament (ACL) injuries, Gait Posture 48 (2016) 165-170.

[12] J.K. Sinclair, H. Vincent, J.D. Richards, Effects of prophylactic knee bracing on knee joint kinetics and kinematics during netball specific movements, Phys. Ther. Sport 23 (2017) 93-98.

[13] C.L. Craig, A.L. Marshall, M. Sjorstrom, A.E. Bauman, M.L. Booth, B.E. Ainsworth, et al., International physical activity questionnaire: 12 -country reliability and validity, Med. Sci. Sports Exerc. 35(8) (2003) 1381-1395.

[14] D.A. Padua, S.W. Marshall, M.C. Boling, C.A. Thigpen, W.E. Garrett, Jr., A.I. Beutler, The Landing Error Scoring System (LESS) is a valid and reliable clinical assessment tool of jump-landing biomechanics: the JUMP-ACL study, Am. J. Sports Med. 37(10) (2009) 1996-2002.

[15] N.A. Saunders, Characteristics of the female landing pattern, University of Ballarat, 2006.

[16] C.M. Laurent, J.M. Green, P.A. Bishop, J. Sjökvist, R.E. Schumacker, M.T. Richardson, et al., A practical approach to monitoring recovery: development of a perceived recovery status scale, J. Strength Cond. Res. 25(3) (2011) 620-628.

[17] A. Cappozzo, F. Catani, U. Della Croce, A. Leardini, Position and orientation in space of bones during movement: anatomical frame definition and determination, Clin. Biomech. 10(4) (1995) 171178.

[18] I. Hanzlíková, J. Richards, K. Hébert-Losier, D. Smékal, The effect of proprioceptive knee bracing on knee stability after Anterior Cruciate Ligament reconstruction, Gait Posture 67 (2019) 242-247. 
[19] E.S. Grood, W.J. Suntay, A joint coordinate system for the clinical description of threedimensional motions: application to the knee, J. Biomech. Eng. 105(2) (1983) 136-144.

[20] J.R. Harry, J.F. Silvernail, J.A. Mercer, J.S. Dufek, Comparison of pre-contact joint kinematics and vertical impulse between vertical jump landings and step-off landings from equal heights, Hum. Movement Science 56 (2017) 88-97.

[21] H. Koga, A. Nakamae, Y. Shima, J. Iwasa, G. Myklebust, L. Engebretsen, et al., Mechanisms for noncontact anterior cruciate ligament injuries: knee joint kinematics in 10 injury situations from female team handball and basketball, Am. J. Sports Med. 38(11) (2010) 2218-2225.

[22] T.K. Koo, M.Y. Li, A guideline of selecting and reporting intraclass correlation coefficients for reliability research, J. Chiropr. Med. 15(2) (2016) 155-163.

[23] T.J. Cleophas, A.H. Zwinderman, Non-parametric tests for Three or more samples (friedman and kruskal-Wallis), Clinical Data Analysis on a Pocket Calculator, Springer2016, pp. 193-197.

[24] C.H. Yeow, P.V.S. Lee, J.C.H. Goh, An investigation of lower extremity energy dissipation strategies during single-leg and double-leg landing based on sagittal and frontal plane biomechanics, Hum. Movement Science 30(3) (2011) 624-635.

[25] O.-E. Olsen, G. Myklebust, L. Engebretsen, R. Bahr, Injury mechanisms for anterior cruciate ligament injuries in team handball: a systematic video analysis, Am. J. Sports Med. 32(4) (2004) 1002-1012.

[26] K.A. Russell, R.M. Palmieri, S.M. Zinder, C.D. Ingersoll, Sex differences in valgus knee angle during a single-leg drop jump, J. Athl. Train. 41(2) (2006) 166-171.

[27] B. Dingenen, B. Malfait, S. Nijs, K.H. Peers, S. Vereecken, S.M. Verschueren, et al., Can twodimensional video analysis during single-leg drop vertical jumps help identify non-contact knee injury risk? A one-year prospective study, Clin. Biomech. 30(8) (2015) 781-787.

[28] N. Karandikar, O.O.O. Vargas, Kinetic chains: a review of the concept and its clinical applications, PM\&R 3(8) (2011) 739-745.

[29] T.E. Hewett, G.D. Myer, The mechanistic connection between the trunk, knee, and anterior cruciate ligament injury, Exerc. Sport Sci. Rev. 39(4) (2011) 161-166. 
Figure 1. Jump-landing task variations.|

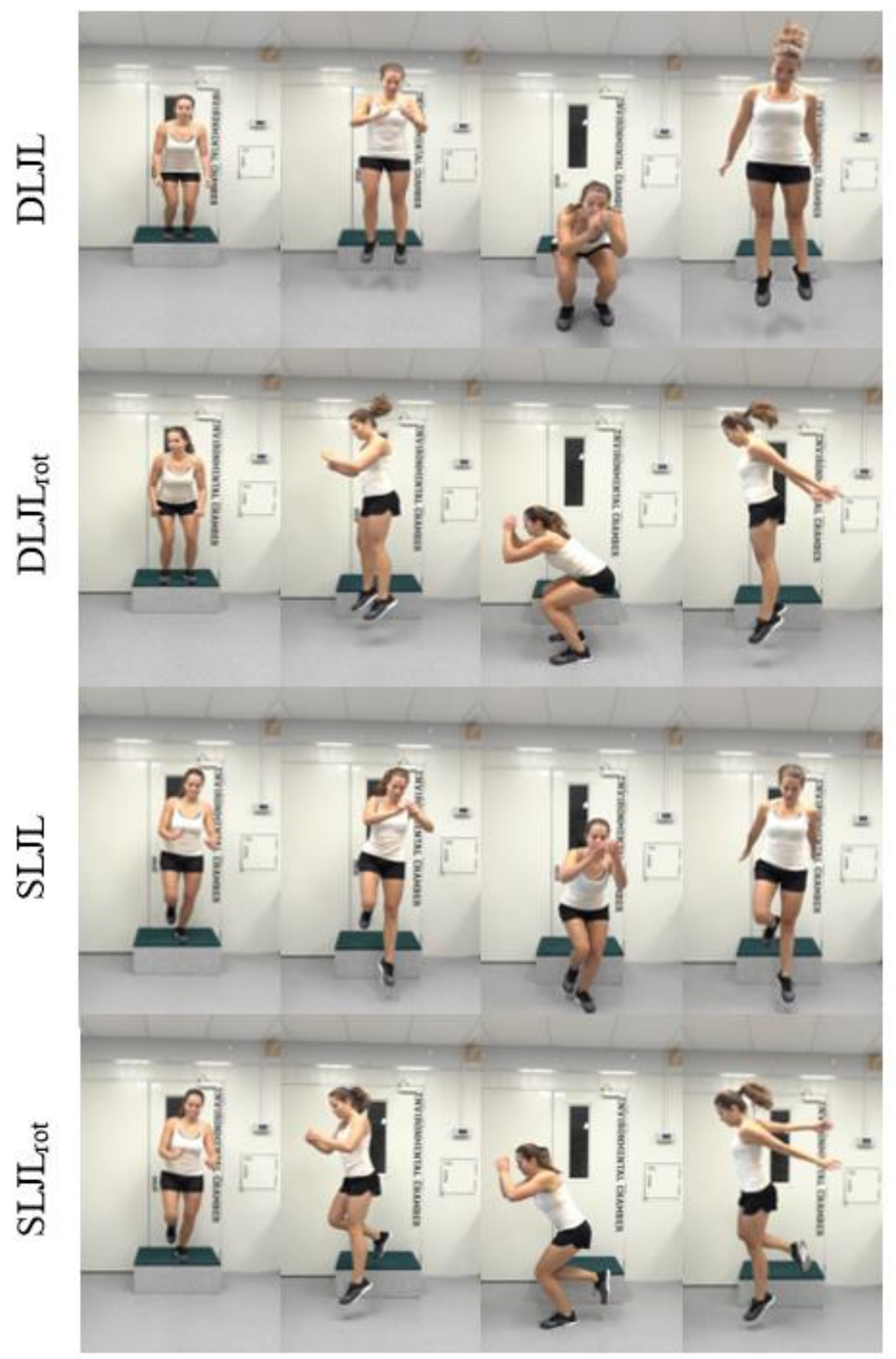

Abbreviations: DLJL, double-leg jump-landing; DLJLrot, rotated double-leg jump-landing; SLJL, single-leg jump-landing; SLJLrot, rotated single-leg jump-landing. 
Figure 2. Marker set.

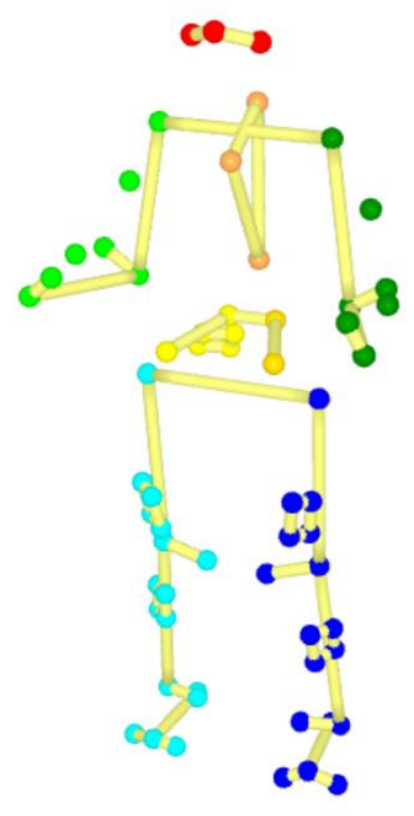

Table 1. Joint movement in all three planes represented by minimum and maximum values.

Sagittal plane $(\mathbf{X})$

Coronal plane (Y)

Transverse plane $(\mathbf{Z})$

\begin{tabular}{lllllll} 
& Minimal values & Maximum values & Minimal values & Maximum values & Minimal values & Maximum values \\
\hline Foot $^{\mathbf{a}}$ & Toe landing & Heel landing & Eversion & Invasion & External rotation & Internal rotation \\
Ankle & Plantar flexion & Dorsiflexion & Abduction & Adduction & External rotation & Internal rotation \\
Knee & Extension & Flexion & Valgus & Varus & External rotation & Internal rotation \\
Hip & Extension & Flexion & Abduction & Adduction & External rotation & Internal rotation \\
Pelvis & Anteversion & Retroversion & ND pelvis drop & D pelvis drop & Rotation to D & Rotation to ND \\
Trunk & Extension & Flexion & Lateral flexion to ND & Lateral flexion to D & Rotation to D & Rotation to ND \\
\hline
\end{tabular}

Abbreviations: $\mathrm{D}$, dominant side; $\mathrm{ND}$, non-dominant side.

${ }^{a}$ Foot-ground angle one frame before initial contact. 
Figure 3. Comparison of intraclass correlation coefficients (ICC) associating (A) values at initial contact (IC), (B) minimal values in the 100 milliseconds (ms) after IC, (C) maximal values in the $100 \mathrm{~ms}$ after IC, and (D) range of motion values in the $100 \mathrm{~ms}$ after IC of biomechanical variables between unanticipated side-step cutting maneuver and jump-landing tasks.

\section{(A) Initial contact}

Comparison of intraclass correlation coefficients (ICC) associating (A) values at initial contact

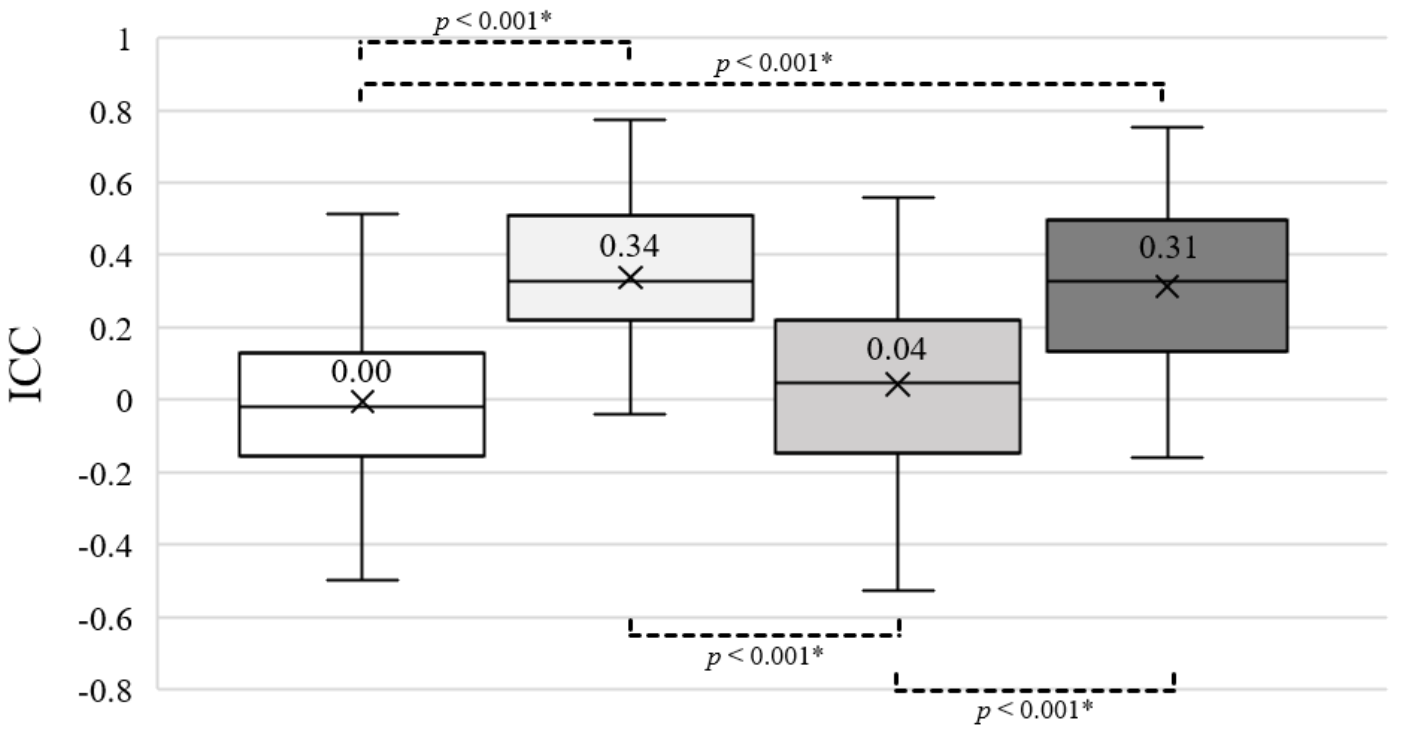

(IC)

Cutting $\sim$ D $\quad$ (B) Minimal values (first 100 ms) $\quad$ tting $\sim$ SLJLrot

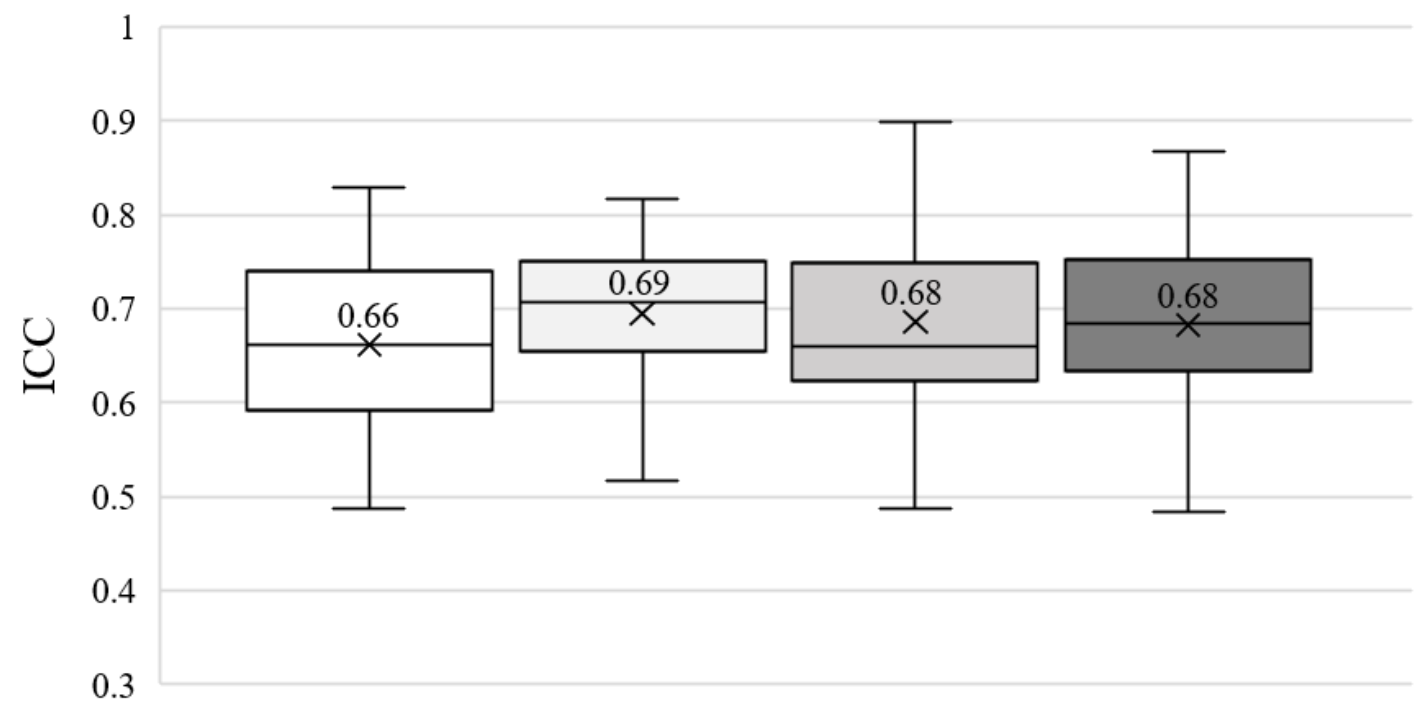

Cutting DLJL $\square$ Cutting DLJLrot $\square$ Cutting SLJL $\square$ Cutting SLJLrot 


\section{(C) Maximal values (first $100 \mathrm{~ms}$ )}

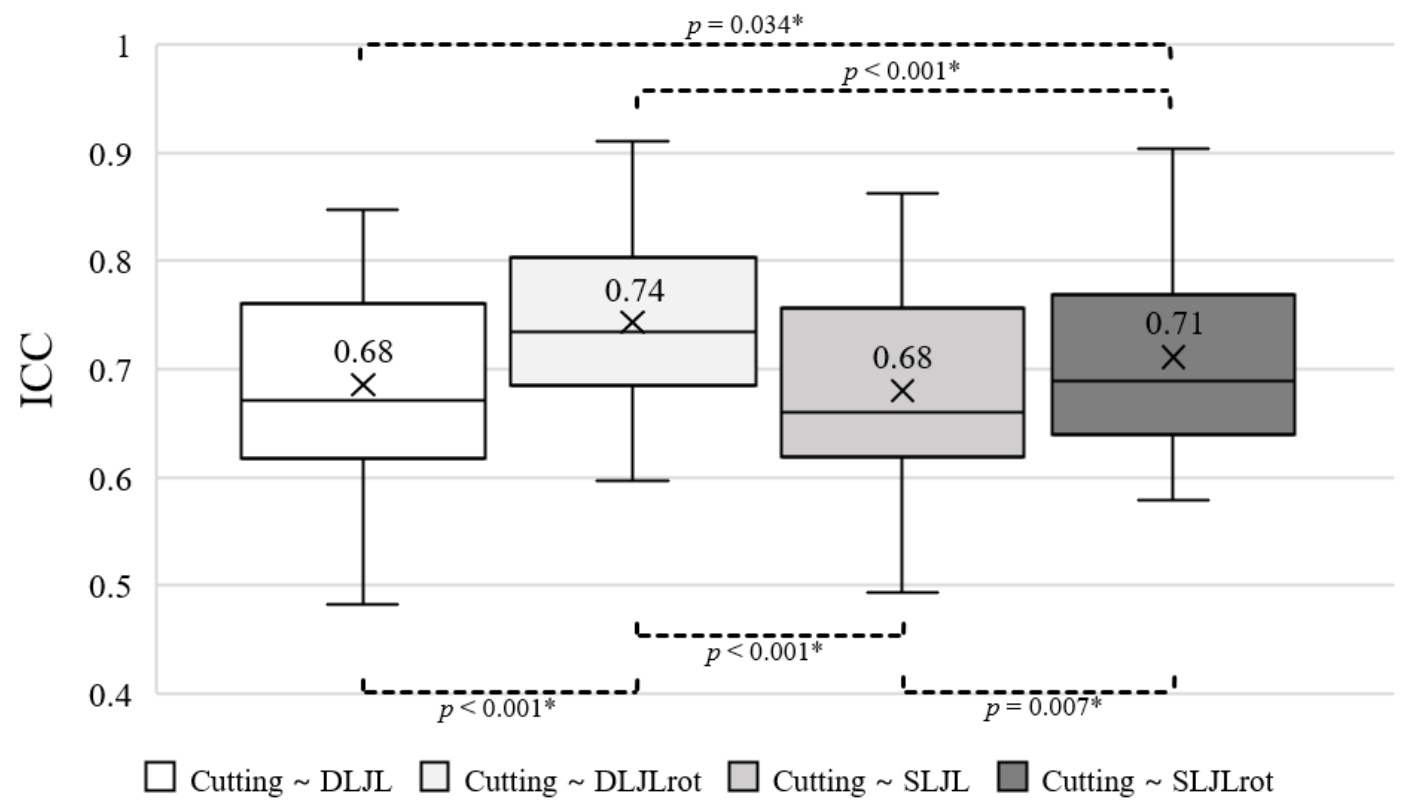

(D) Range of motion values (first $100 \mathrm{~ms}$ )

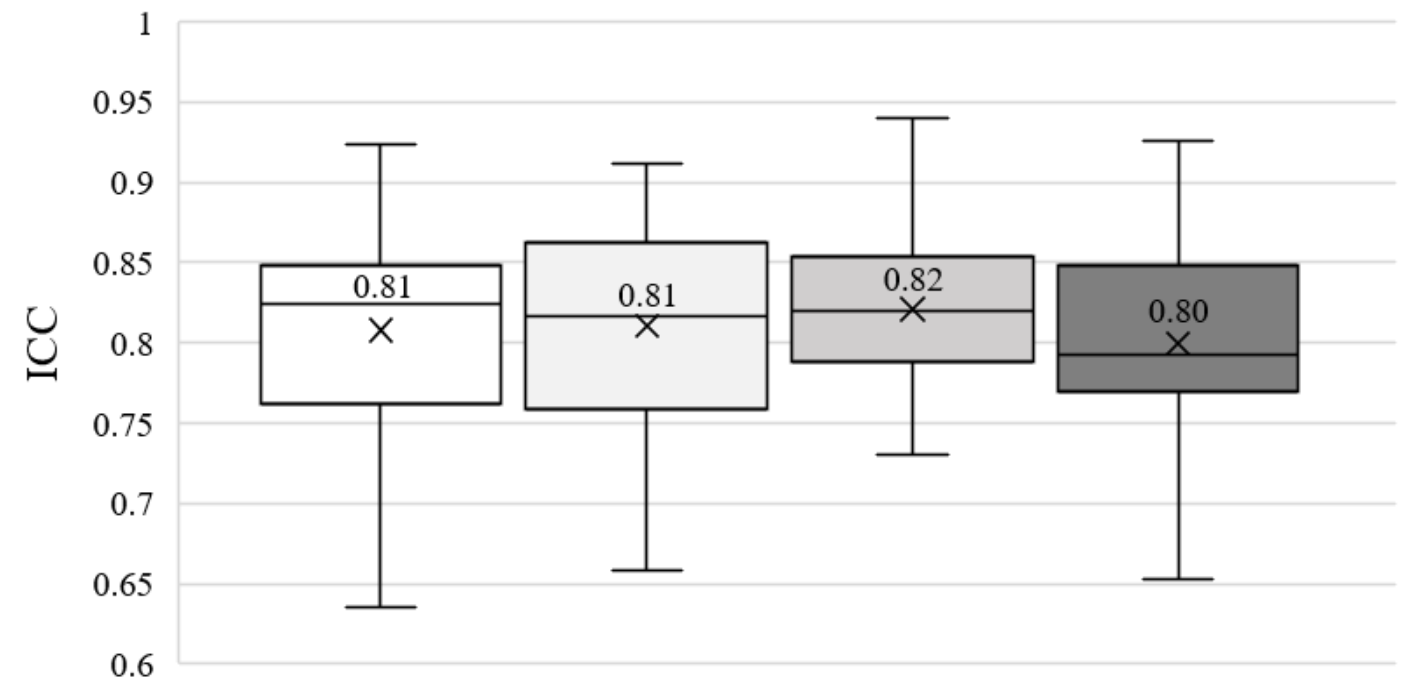

Cutting DLJ $\square$ Cutting DLJrot $\square$ Cutting SLJ $\square$ Cutting SLJrot

Abbreviations: DLJL, double-leg jump-landing; DLJLrot, rotated double-leg jump-landing; SLJL, single-leg jump-landing; SLJLrot, rotated single-leg jump-landing.

Cross indicates the mean value. Horizontal line indicates the median. Error bars represent minimal and maximal values within the sample.

* Indicates significant differences between tasks based on pairwise comparison from Friedman test. 
Figure 4. Subjective ratings of the difficulty of each task.

$$
25
$$

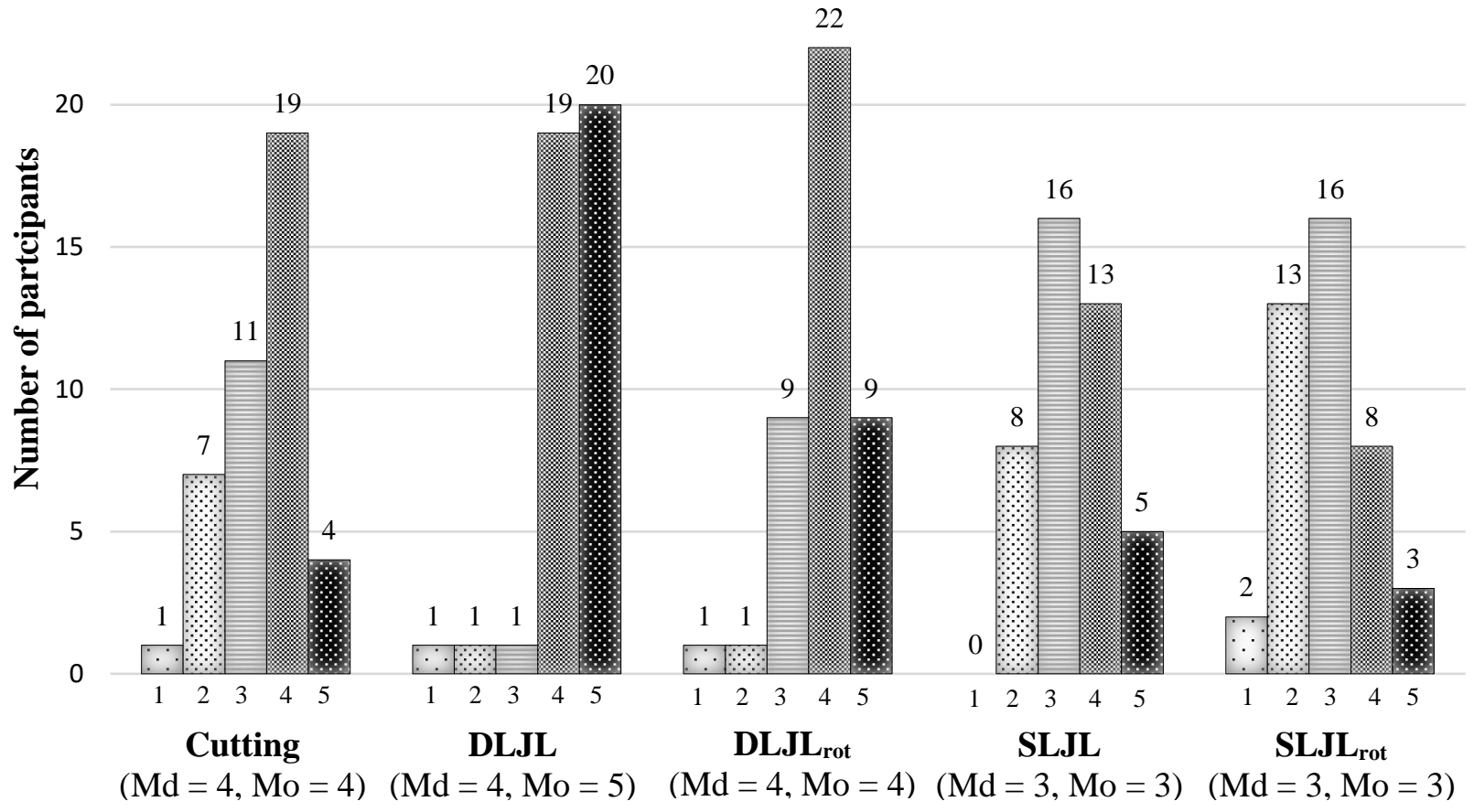

Ratings: 1 , very difficult; 2 , difficult; 3 , neutral; 4 , easy; 5 , very easy

Abbreviations: DLJL, double-leg jump-landing; DLJLrot, rotated double-leg jump-landing; SLJL, single-leg jump-landing; SLJLrot, rotated single-leg jump-landing; Md, median; Mo, mode. 